\title{
Présence au Maroc de la race 2 de Plasmopara halstedii, agent du mildiou du tournesol
}

\author{
EH Achbani ${ }^{1}$, M El Hani ${ }^{1}$, A Douria ${ }^{2}, \mathrm{P}$ Walser ${ }^{3}$, D Tourvieille de Labrouhe ${ }^{3 *}$ \\ 1 Laboratoire de pathologie végétale, CRRASMA, BP 578 Meknès; \\ 2 Département de biologie, faculté des sciences, université IBN-Toufail, Kenitra, Maroc; \\ 3 Station d'amélioration des plantes, Great-Inra, domaine de Crovelle, F-63039 Clermont-Ferrand cedex, France
}

(Reçu le 30 avril 1996 ; accepté le 30 avril 1996)

\begin{abstract}
Résumé - Des tests de laboratoire réalisés sur des isolats marocains de mildiou ont mis en évidence l'existence, en plus de la race 1 dite race "Européenne», d'une race plus virulente qui a le même comportement que la race 2 américaine.
\end{abstract}

Helianthus annuus $=$ tournesol $/$ Plasmopara halstedii $=$ mildiou $/$ race

Summary - Plasmopara halstedii race 2 of downy mildew is present on sunflower in Morocco. The reaction of five different Plasmopara halstedii isolates has been analysed on a range of differential genotypes. Four isolates had a similar reaction to race 1 (the European race). However one isolate had the ability to infect genotypes with no gene of resistance and also the line Rha $266(\mathrm{P} 11)$ which is resistant to race 1. The reaction of this isolate was similar to that of the American race 2. This is the first record of this race in Morocco.

Helianthus annuus $=$ sunflower $/$ Plasmopara halstedii $=$ downy mildew $/$ race

Le mildiou du tournesol (Helianthus annuus L) est dû à Plasmopara halstedii (Farl) Berl et de Toni. C'est un parasite obligatoire qui se présente sous la forme de races physiologiques capables d'attaquer des génotypes de tournesol différents. La résistance est contrôlée par des gènes majeurs appelés $P l$. Actuellement, une dizaine de races et 16 gènes $P /$ ont été décrits (Gulya et al, 1991a, b, 1995). Au Maroc, trois races ont été signalées, la race 1 (Mouzeyar et al, 1993 ; Achbani et Tourvieille, 1993), puis les races 4 et 9 (Gulya et al, 1996).
Cinq souches isolées sur des tournesols malades originaires de différentes régions du Maroc ont été analysées. Le tableau I présente les interactions de ces cinq isolats avec une série de génotypes hôtes différentiels. La contamination des hôtes différentiels et la caractérisation de la résistance sont réalisés selon la méthode décrite par Mouzeyar et al (1994). Après infection, les génotypes sont considérés comme résistants lorsqu'ils ne présentent aucune sporulation sur les organes aériens ou lorsqu'une légère sporulation est visible sur cotylédons mais aucu-

* Correspondance et tirés à part 
Tableau I. Réaction de génotypes de tournesol hôtes différentiels aux isolats marocains de Mildiou, Plasmopara halstedii.

\begin{tabular}{|c|c|c|c|c|c|c|c|c|}
\hline \multirow[t]{2}{*}{$\begin{array}{l}\text { Génotypes } \\
\text { de tournesol }\end{array}$} & \multirow[t]{2}{*}{ Origines } & \multicolumn{2}{|c|}{$\begin{array}{l}\text { Races de } \\
\text { P halstedii a }\end{array}$} & \multicolumn{5}{|c|}{$\begin{array}{l}\text { Isolats marocains } \\
\text { de } \mathrm{P} \text { halstedii }{ }^{a}\end{array}$} \\
\hline & & 1 & 2 & MA1 & MA2 & MA3 & MA4 & MA5 \\
\hline Pérédovic & Population russe & $S$ & $S$ & $\mathrm{~S}$ & $\mathrm{~S}$ & $S$ & $\mathrm{~S}$ & $S$ \\
\hline $\mathrm{Ha} 89$ & Lignée USDA & $S$ & $S$ & $S$ & $S$ & $S$ & $\mathrm{~S}$ & $S$ \\
\hline Rha 266 & Lignée USDA & $\mathrm{R}$ & $\mathrm{S}$ & $\mathrm{R}$ & $\mathrm{R}$ & $\mathrm{R}$ & $\mathrm{R}$ & $\mathrm{S}$ \\
\hline Rha 274 & Lignée USDA & $R$ & $\mathrm{R}$ & $\mathrm{R}$ & $R$ & $\mathrm{R}$ & $\mathrm{R}$ & $\mathrm{R}$ \\
\hline PMI3 & Lignée Inra sélectionnée de DM2 (USDA) & $\mathrm{R}$ & $\mathrm{R}$ & $\mathrm{R}$ & $\mathrm{R}$ & $\mathrm{R}$ & $\mathrm{R}$ & $\mathrm{R}$ \\
\hline HAR5 & Population USDA & $\mathrm{R}$ & $\mathrm{R}$ & $\mathrm{R}$ & $\mathrm{R}$ & $\mathrm{R}$ & $\mathrm{R}$ & $\mathrm{R}$ \\
\hline HA335 & Lignée USDA & $\mathrm{R}$ & $\mathrm{R}$ & $\mathrm{R}$ & $\mathrm{R}$ & $\mathrm{R}$ & $\mathrm{R}$ & $\mathrm{R}$ \\
\hline HA338 & Lignée USDA & $\mathrm{R}$ & $\mathrm{R}$ & $\mathrm{R}$ & $\mathrm{R}$ & $\mathrm{R}$ & $\mathrm{R}$ & $\mathrm{R}$ \\
\hline
\end{tabular}

a $\mathrm{R}$ : résistant; $\mathrm{S}$ : sensible. Les génotypes sont maintenus par autofécondation sous sac à l'Inra, Meknès.

ne sur feuilles. Les génotypes sensibles sporulent sur cotylédons et sur feuilles.

Quatre souches ont un comportement identique à la race 1 , alors que la cinquième apparaît différente des races décrites au Maroc (tableau 1). En effet, l'isolat MA5 sporule sur la lignée Rha 266, possédant le gène $P / 1$ et résistante à la race 1 . Contrairement à la race 4 (Gulya et al, 1996), elle ne sporule pas sur Rha 274 ; de même la race 9 sporule sur DM2 (Gulya et al, 1996) alors que notre isolat ne sporule pas sur PMI3 (lignée tirée de DM2). L'isolat MA5 appartient au pathotype race 2. C'est la première fois que la présence de cette race est décrite au Maroc. L'existence de cette nouvelle race doit être prise en compte dans les programmes de sélection de variétés de tournesol adaptées au Maroc. De même, le comportement, vis-à-vis de toutes les races présentes au Maroc, de l'ensemble des variétés (hybride ou population) actuellement cultivées dans ce pays devrait être analysé. Ceci permettrait de préconiser, pour les variétés sensibles à au moins une race, un traitement de semences avec l'Apron 35SD (métalaxyl) seul anti-mildiou actuellement autorisé au Maroc sur tournesol.

\section{RÉFĖRENCES}

Achbani EH, Tourvieille D (1993) Le tournesol au Maroc, les problèmes phytosanitaires commencent. Phytoma 448, 30-32

Gulya TJ, Miller JF, Viranyi F, Sackston WE (1991a) Proposed internationally standardized methods for race identification of Plasmopara halstedii. Helia 14, 11-20

Gulya TJ, Sackston WE, Viranyi F, Masirevic S, Rashid KY (1991b) New races of the sunflower downy mildew pathogen (Plasmopara halstedii) in Europe and North and South America. $J$ Phytopathol 132, 303-311

Gulya TJ, Viranyi F, Nowell D, Serrhini MN, Arouay K (1996) New races of sunflower downy mildew in Africa. Proc 18th Sunflower Research Workshop, January 11-12, 181-184

Mouzeyar S, Gosset H, El Asri M, Vear F, Tourvieille D (1993) Première description au Maroc du mildiou du tournesol et identification de la race de Plasmopara helianthi. Alawamia 78, 113-128

Mouzeyar S, Walser P, Tourvieille D (1994) Mildiou du tournesol : une nouvelle interprétation du test de résistance. Phytoma 461, 25-26 